\title{
Depressão e qualidade de vida em jovens de 18 a 24 anos no sul do Brasil
}

\author{
Depression and quality of life in young adults aged 18 to 24 years in southern Brazil \\ Mariane Ricardo Acosta Lopez ${ }^{1}$, Juliane Portella Ribeiro ${ }^{2}$, Liliane da Costa Ores ${ }^{3}$, Karen Jansen ${ }^{4}$, Luciano Dias \\ de Mattos Souza ${ }^{4}$, Ricardo Tavares Pinheiro ${ }^{4}$, Ricardo Azevedo da Silva ${ }^{4}$
}

${ }^{1}$ Psicóloga. Mestre. Doutoranda, Programa de Pós-Graduação em Saúde e Comportamento, Universidade Católica de Pelotas (UCPel), Pelotas, RS. ${ }^{2}$ Psicóloga e enfermeira. Mestranda, Programa de Pós-Graduação em Enfermagem, Universidade Federal do Rio Grande (FURG), Rio Grande, RS. ${ }^{3}$ Mestre em Saúde e Comportamento. ${ }^{4}$ Doutor(a). Professor(a), Programa em Saúde e Comportamento, UCPel.

\section{Resumo}

Objetivo: Investigar a associação entre depressão e qualidade de vida em jovens de 18 a 24 anos de idade.

Método: Estudo transversal de base populacional, composto por 1.560 jovens de 18 a 24 anos residentes na zona urbana de Pelotas (RS). A seleção amostral foi realizada por conglomerados: da divisão censitária de 448 setores, 97 foram sorteados aleatoriamente. A avaliação da depressão foi realizada através do Mini-International Neuropsychiatric Interview (MINI), e a qualidade de vida foi mensurada pela Medical Outcomes Study Short-Form General Health Survey (SF-36), ambos validados para uso em língua portuguesa.

Resultados: A prevalência de depressão foi de 12,6\%. A média dos escores de qualidade de vida entre os oito domínios do SF-36 foi menor entre os jovens com depressão, apresentando associação significativa no teste t para todos os domínios $(\mathrm{p}=0,000)$.

Conclusão: Jovens com indicativo de depressão apresentaram menores níveis de qualidade de vida nos domínios explorados.

Descritores: Depressão, qualidade de vida, adulto jovem, estudos transversais.

\begin{abstract}
Objective: To investigate the association between depression and quality of life in young adults aged 18 to 24 years.

Method: This cross-sectional, population-based study included 1,560 young adults aged 18 to 24 years living in the urban area of Pelotas, southern Brazil. Sample selection was based on cluster sampling: of a total of 448 sectors, 97 were randomly selected. Depression was assessed using the Mini-International Neuropsychiatric Interview (MINI), and quality of life was measured using the Medical Outcomes Study Short-Form General Health Survey (SF-36), both validated for use in Brazilian Portuguese.

Results: The prevalence of depression was $12.6 \%$. Mean scores obtained for quality of life in the eight domains assessed by SF-36 were lower in depressive young adults, reaching statistical significance in all domains when assessed using the $t$ test $(p=0.000)$.

Conclusion: Young adults with signs and symptoms suggestive of depression presented lower levels of quality of life based on the domains assessed.

Keywords: Depression, quality of life, young adult, cross-sectional studies.

\section{Introdução}

A depressão se tornou um problema de grande importância nos dias de hoje e parece estar relacionada a uma reação ao mundo moderno. O Relatório Mundial de Saúde ${ }^{1}$ expõe que a urbanização, o envelhecimento e as mudanças globalizadas nos estilos de vida combinam-se entre si para tornar as doenças crônicas e não transmissíveis - incluindo a depressão - causas cada vez mais importantes de morbidade e de mortalidade.

Segundo o Diagnostic and Statistical Manual of Mental Disorders, 4th edition (DSM-IV)2 ${ }^{2}$ o transtorno depressivo maior,

comumente chamado de depressão, caracteriza-se por um período mínimo de 2 semanas durante as quais predomina um humor deprimido ou perda de interesse ou prazer por quase todas as atividades. Em crianças e adolescentes, o humor pode ser irritável ou rabugento em vez de triste. $\mathrm{O}$ indivíduo também pode experimentar pelo menos quatro sintomas adicionais, dentre os quais é possível citar: alteração no apetite, peso, sono e atividade psicomotora; diminuição da energia; sentimentos de desvalia ou culpa; dificuldades para pensar, concentrar-se ou tomar decisões, ou pensamentos recorrentes sobre morte ou ideação suicida, planos ou tentativas de suicídio.
\end{abstract}

Correspondência:

Mariane Lopez, Programa de Pós-Graduação em Saúde e Comportamento, Universidade Católica de Pelotas, Rua Almirante Barroso, 1202, Centro, CEP 96010-280, Pelotas, RS, Brasil. Tel.: (53) 2128.8404. E-mail: mariane_lop@hotmail.com

Não foram declarados conflitos de interesse associados à publicação deste artigo.

Copyright (C) Revista de Psiquiatria do Rio Grande do Sul - APRS

Recebido em 28/06/2010. Aceito em 04/08/2010. 
Existem evidências na literatura internacional de que a depressão acarreta prejuízos à qualidade de vida ${ }^{3-5}$. As pesqui$\operatorname{sas}^{3-7}$ demonstram que existe uma relação inversa entre percepção de qualidade de vida e intensidade de sintomatologia depressiva, ou seja, a percepção de um baixo nível de qualidade de vida é expressa por pacientes com altos níveis de sintomas depressivos. Dessa forma, a melhora ou redução sintomática ofereceria uma melhora direta na qualidade de vida.

Segundo o grupo Quality of Life Assessment da Organização Mundial da Saúde (WHOQOL) ${ }^{8}$, qualidade de vida é a percepção do indivíduo sobre a sua posição na vida, no contexto da cultura e dos sistemas de valores nos quais ele vive e em relação a seus objetivos, expectativas, padrões e preocupações. Essa definição demonstra a importância de explorar as diferentes variáveis que se relacionam com a qualidade de vida - neste caso, o transtorno depressivo.

Optamos por estudar adultos jovens na faixa etária de 18 a 24 anos devido à escassez de pesquisas sobre qualidade de vida e depressão nessa população, visto que a produção existente é voltada para a investigação de adultos, especialmente idosos. A faixa etária sob investigação compreende um período de transição da adolescência para a vida adulta, em que o indivíduo se torna um adulto jovem. A passagem de uma etapa para outra acarreta mudanças, e toda mudança em si mesma pode ser considerada uma crise em busca da nova identidade. Dessa forma, Rubio ${ }^{5}$ lembra que ser adulto jovem está entre os fatores de risco associados à depressão.

Cabe ressaltar que a investigação acerca da associação entre qualidade de vida e depressão em adultos jovens revela-se pertinente e valiosa, uma vez que, até então, essa população não foi alvo de pesquisa no Brasil. Assim, o presente estudo teve por objetivo investigar a associação entre qualidade de vida e depressão em jovens de 18 a 24 anos residentes na zona urbana de Pelotas (RS).

\section{Método}

Foi realizado um estudo transversal, de base populacional, composto por 1.560 jovens selecionados aleatoriamente através de uma amostra por conglomerados. Para tal, dos 448 setores censitários da cidade de Pelotas, 97 foram aleatoriamente incluídos. O tamanho amostral por grupo (com/sem depressão) foi calculado pelo teste $t$ para a comparação entre as médias dos domínios da escala de qualidade de vida Medical Outcomes Study Short-Form General Health Survey (SF-36). A partir da magnitude padronizada de efeito, o domínio aspecto físico apresentou a maior amostra necessária para testar a hipótese de que há diferença entre as médias dos domínios de qualidade de vida da escala SF-36 quando comparados jovens deprimidos e não deprimidos. Sendo assim, o tamanho amostral necessário constatado foi de 176 jovens por grupo. Considerando a hipótese de $13 \%$ para a prevalência de depressão, nosso n amostral mínimo foi de 1.354 jovens de 18 a 24 anos, acrescidos de $15 \%$ para o controle de fatores confundidores.
Os jovens sorteados que aceitaram participar do estudo assinaram um termo de consentimento e, neste momento, foram incluídos na amostra. Já os jovens que não residiam na zona urbana de Pelotas ou que manifestaram incapacidade de compreender ou responder o questionário foram excluídos do estudo. Os instrumentos foram aplicados por alunos do Centro de Ciências da Vida e da Saúde da Universidade Católica de Pelotas (UCPel), previamente treinados para o manuseio da entrevista.

O presente estudo foi aprovado pelo Comitê de Ética em Pesquisa da UCPel, e todos os jovens que tiveram algum transtorno mental diagnosticado durante o estudo foram encaminhados para atendimento no ambulatório de psiquiatria da UCPel.

A depressão foi avaliada utilizando o Mini-International Neuropsychiatric Interview (MINI) ${ }^{8,9}$, uma entrevista diagnóstica padronizada breve, de curta duração (15 a 30 minutos), destinada à utilização na prática clínica e de pesquisa, que visa à classificação diagnóstica dos entrevistados de forma compatível com os critérios do DSM-IV² e da Classificação Internacional de Doenças (CID-10 $)^{10}$. A versão utilizada no presente estudo foi a MINI 5.0 em português ${ }^{9}$, desenvolvida para utilização em cuidados primários e ensaios clínicos. As características psicométricas da entrevista MINI para o diagnóstico de depressão são: sensibilidade de $96 \%$, especificidade de $88 \%$, valor preditivo positivo de $87 \%$, valor preditivo negativo de $97 \%$ e eficiência de $91 \%$.

Para avaliar a qualidade de vida, foi utilizada a escala SF-36. Essa escala é composta por 36 itens que resultam em uma classificação de oito domínios representados por dimensões indicadoras de saúde, a saber: capacidade funcional, aspecto físico, dor, vitalidade, aspecto social, aspecto emocional, saúde mental e estado geral de saúde. Os oito domínios são avaliados em uma escala padronizada de 0-100, na qual um escore mais alto representa um estado melhor de saúde. A escala SF-36 foi adaptada e validada para uso em populações brasileiras por Ciconelli et al. ${ }^{11}$.

Além da entrevista padronizada (MINI) e da SF-36, os jovens responderam ainda a um questionário com as seguintes variáveis: idade, situação socioeconômica, escolaridade, atividade laboral, uso de psicofármacos e abuso de substâncias. Essas variáveis foram avaliadas através do Teste de Triagem do Envolvimento com Álcool, Cigarro e Outras Substâncias (ASSIST) ${ }^{12}$, versão 2.0/0MS, adaptado e validado para o Brasil. A consistência interna do instrumento foi considerada boa, e ele apresentou bons índices de sensibilidade, variando de $84 \%$ a 91, especificidade de 79 a $98 \%$, valores preditivos positivos de 80 a $93 \%$ e negativos de 85 a $96 \%$.

A avaliação econômica dos participantes foi realizada de duas maneiras: através da renda familiar declarada pelos jovens e da aplicação de uma escala de avaliação socioeconômica. Foi utilizada, para essa avaliação, a classificação da Associação Brasileira de Empresas de Pesquisa (ABEP) ${ }^{13}$, que se baseia na acumulação de bens materiais e na escolaridade do chefe da família. Essa classificação enquadra as pessoas em classes (A, B, C, D ou E) a partir dos escores alcançados. A letra $\mathrm{A}$ se refere à classe social mais alta, e a $\mathrm{E}$, à mais baixa. 
Depressão e qualidade de vida em jovens - LOPEZ ET AL.

Para o processamento dos dados, foi realizada dupla entrada dos instrumentos no programa Epi-Info, versão 6.04d. No mesmo software, foi feita uma checagem automática dos dados no momento da digitação, com o uso do programa CHECK (Data Analysis and Statistical Software, College Station, USA), que permitiu a identificação de inconsistências.

A análise dos dados foi realizada utilizando o programa Stata 9 (Centers for Disease Control and Prevention, Atlanta, USA). O teste do qui-quadrado foi utilizado na análise bivariada, visando descrever associações entre depressão e as características da amostra. Já a relação entre depressão e os diferentes domínios de qualidade de vida foi avaliada com o teste $t$. Para ambos os testes, as associações foram consideradas significativas quando $p \leq 0,05$.

\section{Resultados}

Dos 1.762 jovens identificados pela equipe de pesquisa, 202 se recusaram a participar do estudo ou não foram localizados, resultando em uma amostra de 1.560 adultos jovens entrevistados na zona urbana de Pelotas (RS). Em sua maioria, a amostra foi constituída por jovens do sexo feminino $(56,4 \%)$, e $48,1 \%$ pertenciam à classe $C$, segundo a classificação da ABEP. A maior parte dos jovens estava trabalhando (66\%) e não vivia com companheiro $(71,9 \%)$. Em relação à escolarida- de, $46 \%$ estavam estudando ou haviam estudado nos últimos 12 meses, e 54\% não haviam estudado no último ano.

Quanto ao abuso de substâncias, 24,9\% dos jovens faziam uso abusivo de derivados do tabaco e 73,1\% abusavam do consumo de álcool. Ainda, 5,9\% haviam utilizado algum psicofármaco nos últimos 30 dias. Do total da amostra, $13,3 \%$ apresentavam algum transtorno de ansiedade e 7,6\% apresentaram dois ou mais transtornos. O risco de suicídio esteve presente em $8,6 \%$ da amostra.

$\mathrm{Na}$ análise bivariada, as jovens do sexo feminino apresentaram quase 3 vezes maior probabilidade de depressão do que os jovens do sexo masculino $(\mathrm{p}<0,001)$. Não estar estudando no último ano $(\mathrm{p}=0,017)$, ter abusado de álcool e/ou tabaco nos últimos 12 meses $(p<0,001)$ e ter dois ou mais transtornos de ansiedade $(\mathrm{p}<0,001)$ também estiveram significativamente relacionados à depressão. Os jovens com diagnóstico positivo para risco de suicídio apresentaram risco de depressão 8 vezes maior do que os jovens diagnosticados sem risco de suicídio $(\mathrm{p}<0,001)$. Já os jovens que relataram utilizar psicofármacos nos 30 dias anteriores à entrevista apresentaram 3 vezes mais probabilidade de depressão do que os jovens não usuários de psicofármacos nos 30 dias anteriores $(p<0,001)$. Não houve relação estatisticamente significativa entre depressão e as variáveis trabalho $(p=0,340)$ e viver com companheiro $(\mathrm{p}=0,570)$ (Tabela 1$)$.

Tabela 1 - Fatores associados à depressão em jovens de 18 a 24 anos residentes na zona urbana de Pelotas (RS)

\begin{tabular}{|c|c|c|c|c|}
\hline Variável & $\begin{array}{c}\text { Distribuição da amostra } \\
\text { n (\%) }\end{array}$ & $\begin{array}{c}\text { Prevalência de depressão } \\
\text { n (\%) }\end{array}$ & $\begin{array}{c}\text { Razão de prevalência } \\
\text { (IC95\%) }\end{array}$ & $\mathbf{p}$ \\
\hline Sexo & & & & $<0,001$ \\
\hline Feminino & $880(56,4)$ & $144(16,4)$ & $2,14(1,58-2,89)$ & \\
\hline Masculino & $680(43,6)$ & $52(7,6)$ & 1,00 & \\
\hline \multicolumn{5}{|l|}{ Situação socioeconômica } \\
\hline $\mathrm{A}+\mathrm{B}$ & $583(37,4)$ & $54(9,3)$ & 1,00 & 0,001 \\
\hline $\mathrm{C}$ & $751(48,1)$ & $99(13,2)$ & $1,42(1,04-1,95)$ & \\
\hline $\mathrm{D}+\mathrm{E}$ & $226(14,5)$ & $43(19,0)$ & $2,05(1,42-2,97)$ & \\
\hline \multicolumn{5}{|l|}{ Estudando } \\
\hline Sim & $717(46,0)$ & $74(10,3)$ & 1,00 & 0,017 \\
\hline Não & $843(54,0)$ & $122(14,5)$ & $1,40(1,07-1,84)$ & \\
\hline \multicolumn{5}{|l|}{ Vive com companheiro } \\
\hline Sim & $439(28,1)$ & $59(13,4)$ & $1,10(0,83-1,46)$ & 0,570 \\
\hline Não & $1.121(71,9)$ & $137(12,2)$ & 1,00 & \\
\hline \multicolumn{5}{|l|}{ Trabalho } \\
\hline Sim & $1.030(66,0)$ & $123(11,9)$ & 1,00 & 0,340 \\
\hline Não & $530(34,0)$ & $73(13,8)$ & $1,15(0,88-1,51)$ & \\
\hline \multicolumn{5}{|l|}{ Abuso de derivados de tabaco } \\
\hline Sim & $389(24,9)$ & $82(21,1)$ & $2,17(1,67-2,81)$ & $<0,001$ \\
\hline Não & $1.171(75,1)$ & $114(9,7)$ & 1,00 & \\
\hline \multicolumn{5}{|l|}{ Abuso de álcool } \\
\hline Sim & $1.141(73,1)$ & $72(17,2)$ & $1,58(1,21-2,07)$ & 0,001 \\
\hline Não & $419(26,9)$ & $124(10,9)$ & 1,00 & \\
\hline \multicolumn{5}{|l|}{ Transtornos de ansiedade } \\
\hline Sem transtorno & $1.234(79,1)$ & $70(5,7)$ & 1,00 & $<0,001$ \\
\hline Com um transtorno & $207(13,3)$ & $54(26,1)$ & $4,60(3,33-6,35)$ & \\
\hline Com dois ou mais transtornos & $119(7,6)$ & $72(60,5)$ & $10,67(8,14-13,97)$ & \\
\hline \multicolumn{5}{|l|}{ Risco de suicídio } \\
\hline Sim & $134(8,6)$ & $72(53,7)$ & $6,18(4,91-7,78)$ & $<0,001$ \\
\hline Não & $1.426(91,4)$ & $124(8,7)$ & 1,00 & \\
\hline \multicolumn{5}{|l|}{ Uso de psicofármacos } \\
\hline Sim & $91(5,9)$ & $38(41,8)$ & $3,86(2,91-5,13)$ & $<0,001$ \\
\hline Não & $1.460(94,1)$ & $158(10,8)$ & 1,00 & \\
\hline Total & $1.560(100)$ & $196(12,6)$ & - & - \\
\hline
\end{tabular}

IC95\% = intervalo de confiança de 95\%. 
Tabela 2 - Média e desvio padrão dos domínios da escala de qualidade de vida Medical Outcomes Study Short-Form General Health Survey (SF-36) nos grupos de jovens

\begin{tabular}{lcccccc}
\hline Domínios do SF-36 & Total da amostra & $\begin{array}{c}\text { Jovens } \\
\text { com depressão }\end{array}$ & $\begin{array}{c}\text { Jovens } \\
\text { sem depressão }\end{array}$ & $t$ & $\begin{array}{c}\text { Diferença entre } \\
\text { as médias (IC95\%) }\end{array}$ & p \\
\hline Capacidade funcional & $92,2 \pm 12,7$ & $84,2 \pm 17,2$ & $93,4 \pm 11,5$ & 7,29 & $9,24(6,75-11,74)$ & $<0,001$ \\
Aspecto físico & $85,7 \pm 27,5$ & $61,1 \pm 39,0$ & $89,2 \pm 23,5$ & 9,83 & $28,09(22,46-33,72)$ & $<0,001$ \\
Dor & $75,0 \pm 21,5$ & $57,7 \pm 24,2$ & $77,5 \pm 19,5$ & 10,95 & $19,81(16,24-23,37)$ & $<0,001$ \\
Estado geral de saúde & $77,0 \pm 19,8$ & $59,4 \pm 24,5$ & $79,5 \pm 17,6$ & 11,08 & $20,08(16,51-23,65)$ & $<0,001$ \\
Vitalidade & $60,5 \pm 18,4$ & $39,8 \pm 20,0$ & $63,5 \pm 16,1$ & 15,81 & $23,62(20,68-26,56)$ & $<0,001$ \\
Aspecto social & $83,8 \pm 22,2$ & $56,9 \pm 27,8$ & $87,6 \pm 18,3$ & 15,03 & $30,76(26,72-34,79)$ & $<0,001$ \\
Aspecto emocional & $79,2 \pm 34,0$ & $37,7 \pm 41,1$ & $85,1 \pm 28,2$ & 15,62 & $47,39(41,41-53,37)$ & $<0,001$ \\
Saúde mental & $74,0 \pm 20,4$ & $42,5 \pm 20,8$ & $78,5 \pm 15,9$ & 23,49 & $35,98(32,96-39,00)$ & $<0,001$ \\
\hline
\end{tabular}

IC95\% = intervalo de confiança de 95\%.

A prevalência de depressão entre os jovens foi de $12,6 \%$. A média dos escores de qualidade de vida obtidos nos oitos domínios do SF-36 (capacidade funcional, aspecto físico, dor, estado geral de saúde, vitalidade, aspecto social, aspecto emocional e saúde mental) foi menor entre os jovens com depressão, apresentando associação significativa no teste $t$ para todos os domínios ( $\mathrm{p}<0,001)$ (Tabela 2).

Além da associação entre depressão e qualidade de vida, o presente estudo avaliou a qualidade de vida dos participantes que apresentaram depressão com risco de suicídio. Foram encontrados resultados significativos para cinco dos oito domínios do SF-36: estado geral de saúde $(\mathrm{p}<0,001)$, aspecto emocional $(p<0,05)$, saúde mental $(p<0,001)$, aspecto social $(\mathrm{p}<0,001)$ e dor $(\mathrm{p}<0,05)$. Observou-se que os indivíduos com depressão e risco de suicídio apresentaram menor qualidade de vida do que os indivíduos somente com depressão (Figura 1).

\section{Discussão}

Com este estudo transversal, de base populacional, representativo de uma cidade da Região Sul do Brasil, pretendemos avaliar a associação entre depressão e qualidade de vida em jovens, analisando as diferenças entre os sujeitos com e sem depressão, bem como as características daqueles com ideação suicida.

No que diz respeito à análise das características da amostra, a existência de uma maior prevalência de desordens depressivas em sujeitos do sexo feminino reflete as indicações de outros estudos ${ }^{4,14-17}$. Em Lundby (Suécia) ${ }^{18}$, encontrou-se uma incidência anual de depressão de $0,43 \%$ em homens e $0,76 \%$ em mulheres, sendo que as mulheres apresentam maior risco após a puberdade ${ }^{19}$.

Com relação à associação entre depressão e classe social, os achados vão ao encontro de outros estudos que tam-

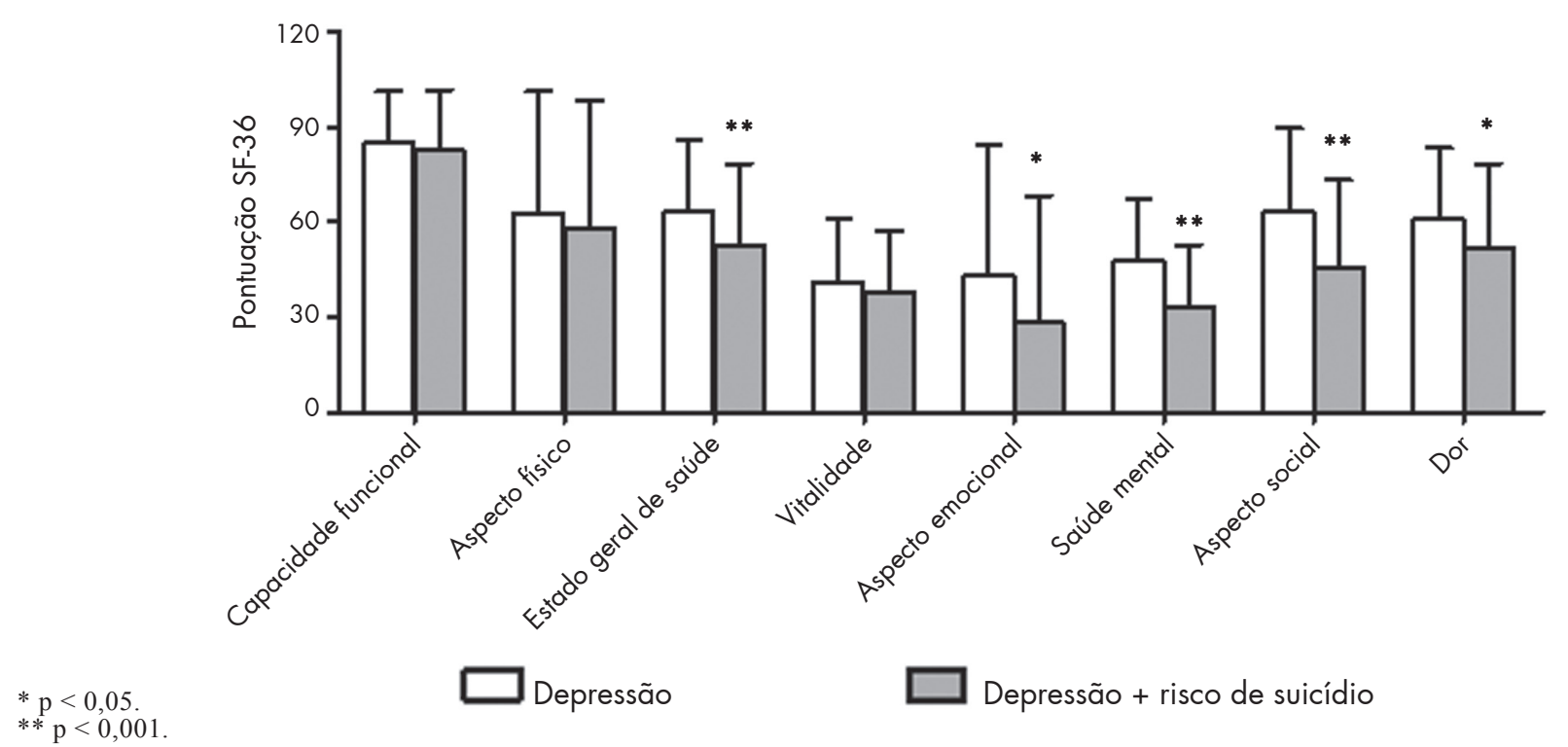

Figura 1 - Associação entre qualidade de vida, depressão e risco de suicídio 
bém observaram um risco maior de problemas de saúde conforme diminui a classe social, evidenciando que os menos favorecidos financeiramente (classes socioeconômicas D e $\mathrm{E}$, segundo a $\mathrm{ABEP}^{13}$ ) apresentam uma prevalência 2 vezes maior de desenvolver o transtorno quando comparados com as demais classes sociais ${ }^{14,16,17,20}$.

De acordo com Sweeting ${ }^{21}$, a diferença entre os gêneros inicia em meados da adolescência. Tal fato pode ser atribuído às diferentes expectativas culturais com relação aos gêneros, visto que, nessa fase, tais expectativas se tornam mais evidentes, acarretando um aumento dos níveis de tensão na transição para a vida adulta ${ }^{15}$.

Da mesma maneira, encontrou-se uma forte associação do abuso de derivados do tabaco e do álcool com a depressão. Esses achados são coerentes com o estudo russo de Averina et al. ${ }^{14}$, embora aqueles autores chamem atenção para o fato de que o aglomerado de problemas associados ao uso abusivo de álcool (efeitos adversos à saúde, problemas no trabalho, nas relações familiares, etc.) pode contribuir para a ocorrência da depressão.

Diferentemente, o fato de viver ou não com um(a) companheiro(a) não apresentou diferenças significativas entre as médias $(\mathrm{p}=0,570)$. No entanto, o estudo de $\mathrm{Lima}^{17}$ demonstrou que a depressão estava mais frequentemente associada a pessoas divorciadas ou separadas, ou em viuvez recente, do que entre solteiros e casados. Seus achados variaram de acordo com o gênero, estando as mulheres solteiras menos suscetíveis à depressão do que as casadas, ao passo que nos homens a situação é inversa.

Surpreendentemente, o fato de estar ou não trabalhando $(p=0,340)$ não resultou em diferenças significativas entre as médias. Esperava-se o oposto, visto que estudos como o da Organização Mundial da Saúde ${ }^{10}$ revelam associação entre situação socioeconômica inativa e depressão. O fato de não estar estudando nos últimos 12 meses apresentou associação significativa com depressão $(\mathrm{p}<0,001)$. Diversos estudos populacionais ${ }^{17}$ sugerem que pessoas com baixa escolaridade apresentam maior prevalência de transtornos mentais. Em um estudo conduzido na cidade de Pelotas $^{22}$, a fração etiológica encontrada na educação foi superior a 50\%, sugerindo que, se fosse proporcionada educação superior à toda população, poder-se-ia reduzir a frequência de transtornos psiquiátricos menores à metade.

A associação existente entre uso de psicofármacos e depressão $(p<0,001)$ sugere que o fato de se ter alguma psicopatologia constitui um fator de risco para depressão. Dessa forma, Brasil ${ }^{23}$ chama atenção para o fato de que um amplo e atualizado conhecimento em psicopatologia e psicofarmacologia e o emprego consciencioso de psicofármacos, associados a outras modalidades de tratamento, podem auxiliar crianças e adolescentes a alcançar uma qualidade de vida melhor.

$\mathrm{O}$ fato de o indivíduo possuir um transtorno de ansiedade aumenta em 4 vezes o risco de desenvolver depressão, e a ocorrência de dois ou mais transtornos aumenta esse risco em 10 vezes, comprovando que a depressão está aumentada em indivíduos com transtornos de ansiedade. Na maioria dos casos, a depressão é secundária a outras doenças psiquiátricas, e os transtornos de ansiedade são os transtornos primários mais comuns ${ }^{24}$.

Igualmente, o risco de suicídio apresentou associação significativa com depressão $(\mathrm{p}<0,001)$. Ladame ${ }^{25}$ lembra que a depressão é um dos problemas psíquicos mais comumente associados ao suicídio em adolescentes e que, ainda que seja o mais frequente, não se pode concluir que seja a causa exclusiva do comportamento suicida.

Os resultados que se referem especificamente à qualidade de vida mostraram uma relação entre queda de todos os aspectos da qualidade de vida e depressão em adultos jovens. Frequentemente, a depressão está associada a reduções importantes na qualidade de vida ${ }^{26,27}$. Similarmente, um estudo que utilizou o SF-36 para medir a qualidade de vida em pacientes depressivos concluiu que os indivíduos depressivos diferiram do grupo não depressivo em todas as escalas ${ }^{28}$.

O caráter pioneiro do presente estudo define a relevância de seus resultados na medida em que abre caminho para futuras investigações sobre a população de adultos jovens e sobre a associação entre depressão e qualidade de vida. Além disso, oferece subsídios para a elaboração de programas de ações preventivas com vistas a ofertar maior qualidade de vida à população.

\section{Conclusão}

Diante do exposto, é possível concluir que a observância da depressão em adultos jovens deve se consolidar como uma variável importante em saúde pública, visto que possui repercussões sobre a qualidade de vida dos indivíduos. Dessa forma, salientamos a importância do diagnóstico precoce e do tratamento adequado da depressão com parte dos cuidados primários de saúde.

\section{Referências}

1. Organização Mundial da Saúde. Cuidados de saúde primários: agora mais do que nunca. Lisboa: OMS; 2008. (Relatório Mundial de Saúde 2008).

2. American Psychiatric Association. Diagnostic and statistical manual of mental disorders, 4th edition (DSM-IV). Washington (DC): APA; 1994.

3. Elliott TE, Renier CM, Palcher JA. Chronic pain, depression, and quality of life: correlations and predictive value of the SF-36. Pain Med. 2003;4(4):331-9.

4. Fleck MP, Lima AF, Louzada S, Schestasky G, Henriques A, Borges VR, et al. Associação entre sintomas depressivos e funcionamento social em cuidados primários à saúde. Rev Saude Publica. 2002;36(4):431-8.

5. Rubio H. Relações entre qualidade de vida e estrutura de personalidade em pessoas deprimidas. PSIC Rev Psicol Vetor Ed. 2002;3(1):58-85.

6. Ay-Woan P, Sarah CP, Lyinn C, Tsyr-Jang C, Ping-Chuan H. Quality of life in depression: predictive models. Qual Life Res. 2006;15(1):39-48.

7. Papakostas GI, Petersen T, Mahal Y, Mischoulon D, Nierenberg AA, Fava M. Quality of life assessments in major depressive disorder: a review of the literature. Gen Hosp Psychiatry. 2004;26(1):13-7.

8. The World Health Organization Quality of Life Assessment (WHOQOL): position paper from the World Health Organization. Soc Sci Med. 1995;41:1403-10. 
9. Amorim P. Mini International Neuropsychiatric Interview (MINI): validação de entrevista breve para diagnóstico de transtornos mentais. Rev Bras Psiquiatr. 2000;22(3):106-15.

10. Organização Mundial da Saúde. Classificação de transtornos mentais e de comportamento da CID-10. Porto Alegre: Artes Médicas; 1993.

11. Ciconelli R, Ferraz M, Santos W, Meinão I, Quaresma M. Tradução para a língua portuguesa e validação do questionário genérico de avaliação de qualidade de vida SF-36 (Brasil SF-36). Rev Bras Reumatol. 1999;39(3):143-50.

12. Henrique IFS, de Micheli D, Lacerda RB, Lacerda LA, Formigoni MLOS. Validação da versão brasileira do Teste de Triagem do Envolvimento com Álcool, Cigarro e Outras Substâncias (ASSIST). Rev Assoc Med Bras. 2004;50(2):199-206.

13. Associação Brasileira de Empresas de Pesquisa (ABEP). Dados com base no Levantamento Socioeconômico do IBOPE, 2003. http://www.ibge.gov.br. Acessado 05/2009.

14. Averina M, Nilssen O, Brenn T, Brox J, Arkhipovsky VL, Kalinin AG. Social and lifestyle determinants of depression, anxiety, sleeping disorders and self-evaluated quality of life in Russia. Soc Psychiatry Psychiatr Epidemiol. 2005;40:511-8.

15. Jorngarden A, Wettergen L, von Essen L. Measuring health-related quality of life in adolescents and young adults: Swedish normative data for the SF-36 and HADS, and the influence of age, gender, and method of administration. Health Qual Life Outcomes. 2006;4(91):1-10.

16. Costa JSD, Menezes AMB, Olinto MTA, Gigante DP, Macedo S, Britto MAP, et al. Prevalência de distúrbios psiquiátricos menores na cidade de Pelotas, RS. Rev Bras Epidemiol. 2002;5(2):164-73.

17. Lima MS. Epidemiologia e impacto social. Rev Bras Psiquiatr. 1999;21(supl 1):1-5.

18. Hagnell O, Lanke J, Rorsman B, Ojesjo L. Are we entering an age of melancholy? Depressive illness in a prospective epidemiological study over 25 years: The Lundby Study, Sweden. Psychol Med 1982;12:279-89.
19. Reppold CT, Hutz CS. Prevalência de indicadores de depressão entre adolescentes no Rio Grande do Sul. Aval Psicol. 2003;2(2):175-84.

20. Mendoza-Sassi R, Béria JU, Fiori N, Bortolotto A. Prevalência de sinais e sintomas, fatores sociodemográficos associados e atitude frente aos sintomas em um centro urbano no Sul do Brasil. Rev Panam Salud Publica. 2006;20(1):22-8.

21. Sweeting H. Reversals of fortune? Sex differences in health in childhood and adolescence. Soc Sci Med. 1995;40:77-90.

22. Lima MS, Béria JU, Tomasi E, Conceição AT, Mari JJ. Stressful life events and minor psychiatric disorders: an estimate of the population attributable fraction in a Brazilian community-based study. Int J Psychiatry Med. 1996;26:213-24.

23. Brasil HHA. Princípios gerais do emprego de psicofármacos. Rev Bras Psiquiatr. 2000;2(supl 2):40-1.

24. Hetem LBA, Graeff FG. Transtornos de ansiedade. São Paulo: Atheneu; 2004.

25. Laudame F. Le suicide chez l'enfant et l'adolescent. In: Lebovici S, Diatkine R, Soule M, eds. Nouveau traité de psychiatrie de l'enfant et l'adolescent. Paris: Presses Universitaires de France; 1995. v. 2, p. 1517-25.

26. Berber JSS, Kupek E, Berber SC. Prevalência de depressão e sua relação com a qualidade de vida em pacientes com síndrome da fibromialgia. Rev Bras Reumatol. $2005 ; 45(2): 47-54$.

27. Xavier FMF, Ferraz MPT, Bertollucci P, Poyares D, Moriguchi EH. Episódio depressivo maior, prevalência e impacto sobre qualidade de vida, sono e cognição em octogenários. Rev Bras Psiquiatr. 2001;23(2):62-70.

28. Kashikar-Zuck S, Vaught MH, Goldschneider KR, Graham TB, Miller JC. Depression, coping and functional disability in juvenile primary fibromyalgia syndrome. J Pain. 2002;3(5):412-9. 